\title{
Fragility and elastic behavior of a supercooled liquid
}

\author{
Madhu Priya and Shankar P. Das \\ School of Physical Sciences, \\ Jawaharlal Nehru University, \\ New Delhi 110067, India.
}

\begin{abstract}
A model for the supercooled liquid is considered by taking into account its solid like properties. We focus on how the long time dynamics is affected due to the coupling between the slowly decaying density fluctuations and the local displacement variables in the frozen liquid. Results from our model agree with the recent observation of Novikov and Sokolov [Nature (London) 431, 961 (2004)] that the fragility index $m$ of a glass forming material is linearly related to the corresponding ratio $K / G$ of the bulk and the shear moduli.
\end{abstract}




\section{INTRODUCTION}

Understanding the basic mechanism for solidification of a supercooled liquid into an amorphous structure constitutes an area of much current research interest in Condensed matter physics. As the liquid is increasingly supercooled its shear viscosity $\eta$ increases and the dynamics slows down enormously. The glass transition temperature $T_{g}$ is characterized by the generic value of $\eta \sim 10^{14} \mathrm{P}$ with the corresponding relaxation time reaching the laboratory time scales. Experiments show that the nature of relaxation in different supercooled liquids approaching the glass transition is not universal. Liquids have been classified as fragile or strong depending on their dynamical behavior in the vicinity of $T_{g}$. Strong liquids show a steady increase of viscosity with the lowering of temperature. In fragile systems the viscosity first increases slowly in the temperature range higher than $T_{g}$ and this trend is followed by a much sharper increase of $\eta$ near $T_{g}$. This classification of different glass forming materials is facilitated in terms of the so called fragility parameter defined [1] as,

$$
m=\partial \ln \eta /\left.\partial\left(T_{g} / T\right)\right|_{T_{g}}
$$

More recently evidence of a dynamic crossover in the value of the viscosity induced by pressure change has also been reported [2]. An associated characteristic of the supercooled state is its solid like behavior [3]. This is manifested in the elastic response of the system to an applied stress. The elastic behavior persists for times shorter than the structural relaxation time. The frozen solid with amorphous structure has transverse sound modes in addition to the longitudinal modes which are present in the normal liquid state. Recently Sokolov and Novikov [4] have demonstrated that the fragility parameter $m$ of a liquid is linearly related to the corresponding ratio $K / G$ of its bulk and shear moduli, i.e., $m-17=29(K / G-1)$. The dependence of the fragility and the vibrational properties of the liquid on the basic interaction potential was also tested recently with computer simulations [5]. This strengthens further the possibility of understanding the vibrational and relaxation properties of the frozen liquid from a common standpoint.

In the supercooled state of the liquid, a tagged particle makes rattling motion and is temporarily trapped in the cage formed by its neighboring particles. The cage eventually breaks giving rise to continuous particle motion. The time for which the tagged particle is localized grows with supercooling. The time correlation function of the collective density fluctuations develops a plateau over intermediate times and eventually decays to zero. Ergodicity thus 
persists in the supercooled liquid over longest time scales. This behavior of the density correlation function is explained in the Mode coupling theory (MCT) [6] of supercooled liquids. The basic theoretical scheme used in this approach involves a formulation of the dynamics in terms of a few slow variables. In this paper we present using the mode coupling approach, a model which includes the solid like properties of a supercooled liquid. We study the effects on the dynamics due to the couplings of slowly decaying density fluctuations with the extra slow modes present in the amorphous solid. Our results conform to the above linear relation between $m$ and $K / G$ reported in Ref. [4]. The paper is organized as follows. In the next section we present the model for the dynamics using an extended set of slow modes for the liquid state. In section III we present the results from the model and demonstrate how it explains the observed data for simple liquids. We end the paper with a discussion.

\section{MODEL STUDIED}

\section{A. Nonlinear dynamics of Slow modes}

The dynamics of the liquid is formulated in terms of a small set of slow modes. The MCT is a basic step in this direction for studying the dynamics of a strongly interacting liquid (at high density) by taking into account the effects of coupling the slow modes in the system. The simplest version of MCT deals with the conserved densities of mass and momentum respectively denoted by $\rho(\mathbf{x}, t)$ and $\mathbf{g}(\mathbf{x}, t)$. We assume the system to be a collection of $N$ classical particles each of mass $\bar{m} . \quad \vec{r}_{\alpha}(t)$ and $\overrightarrow{p_{\alpha}}(t)$ are respectively the position and the momentum of the $\alpha$-th particle at time $t$. For the density $\hat{\rho}$ and the momentum density $\hat{\vec{g}}$ we use the standard prescription [7],

$$
\begin{aligned}
\hat{\rho}(\vec{x}, t) & =\bar{m} \sum_{\alpha=1}^{N} \delta\left(\vec{x}-\overrightarrow{r_{\alpha}}(t)\right) \\
\hat{g}_{i}(\vec{x}, t) & =\sum_{\alpha=1}^{N} p_{\alpha}^{i}(t) \delta\left(\vec{x}-\overrightarrow{r_{\alpha}}(t)\right) .
\end{aligned}
$$

For the solid like state in which the particles vibrate about their mean positions, the above set of slow modes is further extended to include the new collective variable [8] $\mathbf{u}(\mathbf{x}, t)$. This is defined in terms of the displacements $\mathbf{u}_{\alpha}(t)$ of the $\alpha$-th particle, $(\alpha=1$ to $N)$ from their 
respective mean position denoted by $\mathbf{R}_{\alpha}^{o}[9]$ such that $\mathbf{R}_{\alpha}(t)=\mathbf{R}_{\alpha}^{o}+\mathbf{u}_{\alpha}(t)$. We adopt the definition,

$$
\hat{\rho}(\vec{x}, t) \hat{u}_{i}(\vec{x}, t)=\bar{m} \sum_{\alpha=1}^{N} u_{\alpha}^{i}(t) \delta\left(\vec{x}-\overrightarrow{r_{\alpha}}(t)\right),
$$

The metastable positions of the atoms $\mathbf{R}_{\alpha}^{o}(\alpha=1$ to $N)$ in the glassy system remain unaltered for a long time. In a crystal with long range order these positions are independent of time. The equation of motion for $\mathbf{u}$ is obtained using standard procedure [9, 10] in the form of a generalized Langevin equation,

$$
\frac{\partial u_{i}}{\partial t}+\frac{\mathbf{g}}{\rho} \cdot \nabla u_{i}=\frac{g_{i}}{\rho}-\Gamma_{i j} \frac{\delta F}{\delta u_{j}}+f_{i}
$$

where indices $i, j$ refer to the different spatial coordinate axes. The Gaussian noise $f_{i}$ is related to the bare damping matrix $\Gamma_{i j}$, through the fluctuation dissipation relation, $<f_{i}(\mathbf{x}, t) f_{j}\left(\mathbf{x}^{\prime}, t^{\prime}\right)>=2 k_{B} T \Gamma_{i j} \delta\left(\mathbf{x}-\mathbf{x}^{\prime}\right) \delta\left(t-t^{\prime}\right)$, where $k_{B} T=\beta^{-1}$ is the Boltzmann factor. The average thermal

speed of a liquid particle of mass $\bar{m}$ is $v=1 / \sqrt{\beta \bar{m}} . F[\rho, \mathbf{g}, \mathbf{u}]$ is the effective Hamiltonian such that the probability of the equilibrium state is given by $e^{-F}$. For the isotropic solid $F$ is obtained in the general form [9, 11],

$$
F=\int \frac{\mathbf{d x}}{2}\left[\frac{g^{2}}{\rho}+A(\delta \rho)^{2}+2 B \delta \rho s_{T}+K s_{T}^{2}+2 G \tilde{s}_{i j} \tilde{s}_{j i}\right]
$$

where $\delta \rho=\rho-\rho_{o}$ is the density fluctuation and $\rho_{o}$ is the average mass density. The quantities $A$ and $B$ in (5) are related to the static structure factor ( correlation of density fluctuations at equal time ) for the amorphous solid. The symmetric strain tensor field $s_{i j}$ is defined in terms of the gradients of the displacement field $\mathbf{u}(\mathbf{x}), 2 s_{i j}=\left(\nabla_{i} u_{j}+\nabla_{j} u_{i}\right)-\nabla_{i} u_{m} \nabla_{j} u_{m}$ ( such that $s_{i j}=s_{j i}$ ). The trace and the traceless parts of $s_{i j}$, respectively defined as $s_{T}=\sum_{i} s_{i i}$ and $\tilde{s}_{i j}=s_{i j}-\delta_{i j} s_{T} / 3$, appear in the expression (5) for free energy of the isotropic solid.

The equation of motion for $\rho$ is the continuity equation,

$$
\frac{\partial \rho}{\partial t}+\nabla \cdot \mathbf{g}=0
$$

For the momentum density $\mathbf{g}$ the dynamics is given by the generalized Navier-Stokes equation,

$$
\frac{\partial g_{i}}{\partial t}+\sum_{j} \nabla_{j} \sigma_{i j}=\theta_{i}
$$


where $\theta_{i}$ 's are the gaussian noises related to the bare or short time transport properties of the liquid [12]. The symmetric stress tensor $\sigma_{i j}$ is obtained as a sum of the reversible part $\sigma_{i j}^{R}$ and the irreversible (dissipative) part $\sigma_{i j}^{D}$, such that [9]

$$
\sigma_{i j}^{R}=\frac{g_{i} g_{j}}{\rho}+P \delta_{i j}-2 G s_{i j}+2 s_{i j}\left[\bar{K} s_{T}+B \delta \rho\right]+4 G s_{i m} s_{j m}
$$

where $\bar{K}=K-2 G / 3$. The quantity $P$ in (8) is identified with pressure in conventional hydrodynamics and is obtained as local functional of the hydrodynamic fluctuations,

$$
P=\left(A \rho_{o}-B\right) \delta \rho+\left(B \rho_{o}-\bar{K}\right) s_{T}+A \frac{\delta \rho^{2}}{2}-\bar{K} \frac{s_{T}^{2}}{2}-G s_{l m} s_{m l} .
$$

The dissipative part of the stress tensor $\sigma_{i j}^{D}$ is expressed in terms of the bare viscosities.

$$
\sigma_{i j}^{D}=-\eta_{0}\left[\nabla_{i} v_{j}+\nabla_{j} v_{i}-\frac{2}{3} \delta_{i j}(\nabla \cdot \mathbf{v})\right]-\zeta_{0} \delta_{i j}(\nabla \cdot \mathbf{v})
$$

where $\eta_{0}$ and $\zeta_{0}$ are the bare shear and bare bulk viscosities respectively and $\mathbf{v} \equiv \mathbf{g} / \rho$.

\section{B. Renormalization of transport coefficients}

The time correlation functions of the slow modes are obtained by averaging over the

noises in the nonlinear equations of motion using standard field theoretic methods [13]. The correlation functions between two slow modes $\psi_{\alpha}$ and $\psi_{\beta}$ are defined as,

$$
G_{\alpha \beta}(12)=<\psi_{\alpha}(1) \psi_{\beta}(2)>
$$

The dispersion relations for the various hydrodynamic modes in the system are obtained from the pole structures of the correlation functions. The corrections to the correlation functions due to the nonlinearities in the equations of motion for the slow variables are expressed in terms of the self-energy $\Sigma$ defined through the Dyson equation

$$
G^{-1}(\mathbf{q}, \omega)=\left[G^{0}(\mathbf{q}, \omega)\right]^{-1}-\Sigma(\mathbf{q}, \omega)
$$

$G^{0}(\mathbf{q}, \omega)$ refers to the matrix of the correlation functions corresponding to the linear dynamics of the fluctuations. Assuming the amorphous solid state to be isotropic the correlation function can be separated in terms of a longitudinal and a transverse part as

$$
G_{\alpha_{i} \beta_{j}}(\mathbf{q}, \omega)=\hat{q}_{i} \hat{q}_{j} G_{\alpha \beta}^{L}(\mathbf{q}, \omega)+\left(\delta_{i j}-\hat{q}_{i} \hat{q}_{j}\right) G_{\alpha \beta}^{T}(\mathbf{q}, \omega)
$$


Similarly the self-energies are split into their respective longitudinal and transverse parts denoted by $\Sigma_{L}$ and $\Sigma_{T}$. Using the Dyson equation (12) the renormalized viscosity of the liquid taking into account the nonlinearities in the dynamics is obtained in the form

$$
\Gamma(\mathbf{q}, \omega)=\Gamma_{0}+\left[\frac{1}{2 k_{B} T}\right] \gamma_{\hat{g} \hat{g}}^{L}(\mathbf{q}, \omega)
$$

where

$$
\Sigma_{\hat{g} \hat{g}}^{T, L}(\mathbf{q}, \omega)=-q^{2} \gamma_{\hat{g} \hat{g}}^{T, L}(\mathbf{q}, \omega)
$$

The functional forms of the self energies are computed with the diagrammatic techniques of the MSR field theory [14]. Of particular interest in the context of glassy dynamics is the density auto correlation function $\phi(q, t)$. The Laplace transform of $\phi(q, t)$ (which is normalized with respect to its equal time value) is obtained as a two step continued fraction in terms of the memory function $\Gamma(q, z)[12]$,

$$
\phi(q, z)=\frac{1}{z-\Omega_{o}^{2} /[z+\Gamma(q, z)]} .
$$

$\Omega_{0}$ is the microscopic frequency of the liquid state[15]. For the case of a normal liquid with only the standard set of conserved densities as a slow variable, the dominant nonlinearity in the momentum equation comes from the coupling of the density fluctuations in the Pressure functional in (8). This obtains [16] the standard mode coupling model in which memory function includes only products of density correlation functions [17, 18]. The present formulation involving an extended set of hydrodynamic variables which include $\mathbf{u}$ ( in addition to the conserved densities) obtains the longitudinal as well as the transverse sound modes in the amorphous solid. Their speeds are respectively obtained as, $c_{L}^{2}=M / \rho_{o}+A \rho_{o}-2 B$ and $c_{T}^{2}=G / \rho_{o}[11]$, where $M=K+4 G / 3$ is the longitudinal modulus. By including the coupling of density fluctuations $\delta \rho$ with the displacement field $\mathbf{u}$ ( see eqn. (8)), a new contribution to the memory function $\Gamma(q, t)$ is obtained. At the one loop level this is obtained as,

$$
\Gamma(\mathbf{q}, \omega)=\Gamma_{0}+\Gamma^{(1)}(\mathbf{q}, \omega)+\Gamma^{(2)}(\mathbf{q}, \omega)
$$

$\Gamma^{(1)}(\mathbf{q}, \omega)$ and $\Gamma^{(2)}(\mathbf{q}, \omega)$ are given by the expressions

$$
\Gamma^{(1)}(\mathbf{q}, \omega)=\frac{8 B^{2}}{k_{B} T} \int \frac{d^{3} \mathbf{p}}{(2 \pi)^{3}} \int \frac{d \Omega}{2 \pi} p^{2}\left[u^{4} G_{u u}^{L}(\mathbf{p}, \Omega)+u^{2}\left(1-u^{2}\right) G_{u u}^{T}(\mathbf{p}, \Omega)\right] G_{\rho \rho}(\mathbf{q}-\mathbf{p}, \omega-\Omega)
$$


and

$$
\Gamma^{(2)}(\mathbf{q}, \omega)=\frac{A^{2}}{k_{B} T} \int \frac{d^{3} \mathbf{k}}{(2 \pi)^{3}} \int \frac{d \Omega}{2 \pi} G_{\rho \rho}(\mathbf{k}, \omega) G_{\rho \rho}(\mathbf{q}-\mathbf{k}, \omega-\Omega)
$$

The role of convective nonlinearities is assumed to be absorbed in $\Gamma_{0}$ which is the bare part or short time part of the transport coefficients. The contribution $\Gamma^{(1)}$ is obtained from the first diagram of fig. 1 resulting from the coupling of the displacement field $\mathbf{u}$ with the density fluctuation $\delta \rho . \Gamma^{(2)}$ is the contribution from the second diagram in fig. 1 coming from the coupling of density fluctuations. In evaluating these diagrammatic contributions we make the approximation that for time scales ( short compared to the structural relaxations) over which the supercooled liquid displays elastic behavior, the longitudinal and transverse correlations of the displacement field are frozen ( constant in time), i.e., $\phi_{u u}^{L, T} \sim \delta(\omega)$. Therefore as a result of the solid like behavior over intermediate time scales, $G_{u u}^{L}$ and $G_{u u}^{T}$ are then obtained as

$$
G_{u u}^{L}(\mathbf{k}, t)=\frac{k_{B} T}{M k^{2}}, \quad G_{u u}^{T}(\mathbf{k}, t)=\frac{k_{B} T}{G k^{2}}
$$

where the $k^{-2}$ dependence of the correlation function arises from the static structure factor for the displacement fields. Substituting these approximate forms for the displacement correlations and evaluating the integrals in (18) and (19), we obtain for the density correlation function and the memory function the simple form of coupled nonlinear integral equations,

$$
\begin{aligned}
\phi(z) & =\frac{1}{z-\Omega_{o}^{2} /[z+\Gamma(z)]} \\
\Gamma(t) & =c_{1} \phi(t)+c_{2} \phi^{2}(t)
\end{aligned}
$$

where in eqns. (21) and (22) we have dropped the wave vector dependence of $\phi$ and $\Gamma$ for simplicity. The density correlation function is approximated in the form $G_{\rho \rho}(q, t)=$ $\chi_{\rho \rho}(q) \phi(t)$ with $\chi_{\rho \rho}$ being the equal time correlation of the density correlation function determined by the thermodynamic properties like temperature and density. The constants $c_{1}$ and $c_{2}$ in (22) are determined from an evaluation of the vertex functions in this approximation ( of wave vector independence) as,

$$
c_{1}=\frac{8}{3}\left(\frac{\lambda_{0} \Delta_{\sigma}}{1-\Delta_{\sigma}}\right)[1+f(\sigma)], \quad c_{2}=\frac{\lambda_{o}}{\left(1-\Delta_{\sigma}\right)^{2}}
$$


where $\Delta_{\sigma}=\Delta_{o}(1-2 \sigma) /(2-2 \sigma)$, and $f(\sigma)=2 / 5(1-2 \sigma)$ with $\sigma$ being the Poisson's ratio $\sigma=(3 K-2 G) /[2(3 K+G)]$. We have used in the expressions (23) the definitions $\Delta_{o}=B^{2} /(A G)$ and $\lambda_{o}=\left(\Lambda^{3} / 6 \pi^{2} n_{o}\right)\left(v / c_{L}\right)^{2}$ in terms of parameters dependent on the thermodynamic state of the system. The equilibrium number density of particles in the fluid is $n_{o}\left(\rho_{o}=\bar{m} n_{o}\right) . \Lambda$ is the upper cutoff of wave vector representing the shortest length up to which the fluctuations are considered. In considering the mode coupling expression for the viscosity we have ignored here the presence of very slow vacancy diffusion mode and its coupling to density fluctuations. Finally, it is useful to note here that we are considering the simple form of the model in which all processes giving rise to ergodic behavior in the asymptotic dynamics have been ignored.

\section{RESULTS}

The central focus in the present analysis is the time dependence of the density autocorrelation function $\phi(t)$. From the coupled set of eqns. (16) (inverse Laplace transformed in the time space) and (22), we obtain a nonlinear integro-differential equation for the dynamics of $\phi(t)$,

$$
\ddot{\phi}(t)+\dot{\phi}(t)+\phi(t)+\int_{0}^{t} d s \Gamma[\phi(t-s)] \dot{\phi}(s)=0
$$

where the dots refer to derivative with respect to time. This equation is solved numerically to obtain the behavior of the density correlation function. It is clear that the dynamics of the density correlation function $\phi(t)$ is driven here by the memory function $\Gamma(t)$ which is expressed in terms of $\phi$ itself. This constitutes a nonlinear feed back mechanism [17, 18] causing a dynamic transition of the liquid to a nonergodic state in which the long time limit of the density correlation function $\phi(t \rightarrow \infty)=f$. The quantity $f$ is termed as the non ergodicity parameter (NEP). In the plane of $c_{1}$ and $c_{2}$ the dynamic transition occurs along the line $c_{1}=2 \sqrt{c_{2}}-c_{2}\lfloor 19]$.

In fig. 2 we display the phase diagram with $c_{1}$ and $c_{2}$ showing the ideal glass transition line. Though this dynamic transition is finally cutoff due to ergodicity restoring mechanisms, it marks a cross over in the dynamical behavior of supercooled liquid. The liquid state is characterized by the density correlation function following initial power law relaxations, followed by a final stretched exponential decay $\phi \sim \exp \left[-(t / \tau)^{\beta}\right][19$, 20]. In the glassy state the density correlation function decays to a nonzero value equal to the non-ergodicity 
parameter. In fig. 2 we have also shown the values of $c_{1}$ and $c_{2}$ corresponding to the nonergodic states in which the dynamics is studied in this paper (to be explained below). For presenting our results in the following, we express time in units of $\Gamma_{o} /\left(\rho_{o} c_{L}^{2}\right)$ where $\Gamma_{o}=\zeta_{o}+4 \eta_{o} / 3$ is the bare longitudinal viscosity of the liquid related to its short time dynamics. We keep the $\lambda_{o}$ appearing in the expression (23) for the coupling constants of the memory function fixed at a constant value $(=.4)$ throughout the calculation. This numerical value is reached by treating $\lambda_{o}$ as an adjustable parameter here for comparison of the results of the present model with experimental data. This essentially implies that the cutoff $\Lambda$ of wave vector integration is being treated as an adjustable parameter in the coarse grained model we present here.

A key quantity of interest in the present analysis is the fragility parameter $m$ which by definition is related to the final relaxation behavior of the supercooled liquid near $T_{g}$. But over this temperature range, the MCT approach has not been very successfull[6] in explaining the relaxation behavior. A useful observation[4] in this respect is that the slope of the viscosity vs. inverse-temperature plot in the high temperature range can be linked with the fragility. This is justified as follows : For very fragile systems (large $m$ ) the slope of the $\eta$ vs. $T_{g} / T$ curve at temperatures near $T_{g}$ is large and hence it must be correspondingly small at the other end of the Angell plot, i.e., for temperatures much higher than $T_{g}$. This is because the curves for different $m$ meet at both ends on the $T_{g} / T$ axis. By examining experimental data, it was pointed out [4] that the slope of the $\eta$ vs. $T_{g} / T$ curve on the high temperature side is inversely related to the fragility $m$. Since MCT is a valid theory for the slow dynamics well above $T_{g}$, we have investigated the relaxation behavior which follows from the present model in this high temperature range. We focus on the growth of the relaxation time $\tau$ instead of that of the viscosity $\eta$. In our model, increasing the parameter $\Delta_{o}$ brings the system closer to the ideal transition ( since it results in an increase of $\tau$ ) and hence this parameter is treated like the inverse of temperature, $\Delta_{o} \propto T_{g} / T$. The dependence of the relaxation time $\tau$ on $\Delta_{o}$ at various (fixed) $K / G$ values is displayed in fig. 3. For large $K / G$ the observed variation is similar to the $\eta$ vs. $T_{g} / T$ curve of a fragile liquid. Although according to MCT model the power law behavior describes the relaxation time data in this range better, we fit $\tau$, following Ref. [4], to an Arrhenius form with activation energy $\kappa$, i.e., $\tau \sim \exp \left(\kappa \Delta_{o}\right)$. By the property of the Angell plot referred to above, we assume $\tilde{m}=1 / \kappa$ to be proportional to the fragility $m . \Delta_{o}$ is proportional to $T_{g} / T$ with 
the proportionality constant being independent of $K / G$. Thus we assume that constants $A$ and $B$ in the expression (5) for the free energy are only functions of temperature and the two elastic constants $K$ and $G$ are to be treated as independent entities. Fig. 4 displays $\tilde{m}$ against the variation of $K / G$ showing an approximately linear behavior. Therefore the present model agrees qualitatively with the linear correlation predicted by Novikov and Sokolov. It should be noted that the inverse nature of the relations between fragility and the slope of the viscosity vs. inverse-temperature plot respectively at the high and low temperature sides is a property of the Angell plot itself. However the reciprocal relation i.e. $\kappa \propto 1 / m$ as used above can only be justified in a qualitative manner. In order to demonstrate the sensitivity of the results on the values of the parameters $\Delta_{o}$ and $\lambda_{o}$ we display in fig 4 . the $\tilde{m}$ vs $K / G$ curves for different values of $\lambda_{0}$ ranging from $\lambda_{0}=0.3$ to $\lambda_{0}=0.8$. The nature of the curves are qualitatively similar to what the best fit value for $\lambda_{o}$ obtains.

Next we consider the dynamics on the other side of the transition when the density correlation function freezes to a constant until ergodicity restoring processes take over. In the glassy state, the NEP $f$ is estimated from the plateau which $\phi(t)$ reaches over long time. Fig. 5 displays the variation of $f$ with the ratio $K / G$. The nonergodic behavior and the elastic response of the supercooled liquid as seen here are valid over initial time scales for which the system is frozen and is solid like. However estimating the fragility parameter $m$ directly involves computing the temperature dependence of viscosity or relaxation time near $T_{g}$. As already pointed out, the long time dynamics in the deeply supercooled state close to $T_{g}$ is still beyond the scope of MCT. Our calculation of the NEP at low temperatures here only refer to intermediate time scales up to which the present description in terms of the supercooled liquid is valid. Therefore in order to link fragility $m$ with the elastic properties of the supercooled liquid we make use of the dependence of the fragility $m$ on the NEP $f$ as inferred from analysis of experimental data [21]. In fig. 6 the data points and best fit curve for the experimental results linking NEP $f$ with the fragility $m$, i.e., $m=167-176 f$, is shown. The NEP values noted here are taken at the glass transition temperature $T_{g}$ [21]. To test this empirical relation further we compare the $\alpha_{o}=(1-f) / f$ vs. $m$ in the inset of fig. 6 with another set of experimental data [22] from X-ray Brillouin scattering. Similar qualitative behavior is seen validating the link between the NEP with the fragility. The dependence of $m$ on the ratio $K / G$ of elastic constants is then obtained through their 
common dependence on the NEP $f$. The variation of $m$ with $K / G$ is shown in fig. 7 . We also display the experimental data (from which the linear relation of Ref. [21] was proposed) in the same figure, showing reasonable agreement with the predictions of the present model. In reaching this agreement between the theoretical model and experimental data we adjust the temperature dependent parameter $\Delta_{o}$ to the value 1.7. As argued above $\Delta_{o}$ is proportional to $T_{g} / T$ and hence if we assume near $T_{g}, \Delta_{o}=c_{o}\left(T_{g} / T\right)$, then $c_{o}=1.7$ obtains the best fit of the present model's predictions with results of Ref. [4]. To demonstrate the sensitivity of the results on the values of the adjusted parameters we display in fig 7 . ( as in fig 4 for $\tilde{m}$ ) the fragility $m$ vs $K / G$ curve for different values of $\lambda_{0}$ ranging from $\lambda_{0}=0.3$ to $\lambda_{0}=0.8$. $\Delta_{0}$ value is kept same for all the curves i.e. $\Delta_{0}=1.7$. Usually fragility index measurements refer to the slope of the viscosity vs. inverse temperature plot at the calorimetric glass transition temperature $T_{g}$. Hence the parameter $\Delta_{o}$ has been kept fixed here. The roughly linear behavior of the $m$ vs. $K / G$ curve is retained over the whole range of the value of the parameter $\lambda_{0}$. The variations of $f$ and $m$ with $K / G$ as displayed in the fig. 5 and 7 respectively are obtained at this same fixed value for $\Delta_{o}$. With the variation of $K / G$, the corresponding values of $c_{1}$ and $c_{2}$ are changed as shown by filled circles in the fig. 2. With increasing value of $K / G$ we move to regions of small values of the couplings $c_{1}$ and $c_{2}$.

Finally we make two predictions using the proposed model linking the elastic behavior with relaxation properties in the glassy systems. According to the present model the density correlation function $\phi(t)$ has a complex relaxation behavior. On the ergodic side of the transition line, the initial power law relaxations change over to a stretched exponential decay with a stretching exponent $\beta$. As a further illustration of the present model, we display in fig. 8 the variation of the stretching exponent $\beta$ with the Poisson's ratio $\sigma=$ $(3 K-2 G) /[2(3 K+G)]$. For the results shown here we keep $\Delta_{o}$ fixed at a typical liquid state value $\Delta_{0}=0.885$. It should be noted that following the discussion above the $\beta$ presented here corresponds to temperatures much above $T_{g}$. On the other hand for computing the fragility $m$ vs. Poisson's ratio $\sigma$ in terms of the non ergodicity parameter $f$, as described above in Fig. 5-7, we work on the nonergodic side of the transition line , i.e. in the glassy phase where the freezing of the density correlation function is clearly visible $\left(\Delta_{o}=1.7\right)$. We do not have in the present mode coupling model a way of computing the $\beta$ in this low temperature regime which will require taking into account the role of ergodicity restoring mechanisms in the long time dynamics. Hence the predicted behavior of the stretching 
exponent should be tested at higher temperatures away from $T_{g}$.

Another suitable quantity is the power law exponent of relaxation near $T_{g}$. On the nonergodic side of transition line, the system is dynamically frozen initially until the ergodicity restoring processes take over. For initial time scales ( beyond the microscopic times) the density correlation function develops a plateau $f$ with a power law form $\phi(t)=f+C t^{-a}$ decay. From the present model we are able to predict the dependence of the power law exponent $a$ on the $K / G$ ratio. Fig. 9 displays the dependence of the exponent $a$ on $K / G$. The inset shows the nature of the power law behavior at a particular value of $K / G=2.6$. The curve is obtained at $\Delta_{0}=1.7$. According to the predictions of the present model the power law exponent grows sharply for less fragile systems indicating a sharper freezing of the system over intermediate time scales for strong liquids.

\section{DISCUSSION}

The present model for the amorphous system is constructed in terms of an extended set of slow modes. The approximations which go into the present formulation of the dynamics for the solid like state are well known and standard. In order to define the slow variable which accounts for the solid like nature of the amorphous state, we require reference to a rigid lattice. On the other hand the ergodicity restoring process in the system invalidates the existence of any such rigid structure over longest time scales. Therefore the elastic description of the liquid is only valid up to the time scale of structural relaxation. A selfconsistent treatment with dynamic connections between the elastic and viscous behavior of the system will possibly provide a way to make a unified description including both of the above two aspects of the glassy state. Furthermore, the atoms in the solid vibrate around the random lattice sites over intermediate time scales. Generally strong glassy systems have a higher network forming tendency than the highly fragile systems [23]. In the latter structural degradation occurs more easily. Thus for systems with very high $m$ values the underlying assumptions of the present model hold for shorter time scales.

The present model which considers the relation of the fragility of the liquid to its elastic properties brings out some basic characteristics of the dynamics. In fig. 7 we se that the increase in the fragility $m$ corresponds to higher values of the ratio $K / G$ while from fig. 2 it follows that coupling $c_{1}$ and $c_{2}$ between the slow modes is weaker for larger values of this 
ratio. Therefore the trend of the experimental data indicates that in a more fragile system there is a weaker coupling of the hydrodynamic modes. This is particularly relevant since the coupling between the slow modes constitutes (through the formulation of the mode coupling theories ) the basic mechanism for slow dynamics in supercooled liquids. Also, since it follows from the present theory that larger values of $K / G$ give rise to lower values of the nonergodicity parameter, the strength of the freezing or jamming of the system is weaker in more fragile systems.

It is important to note that the present model is formulated for the case of simple liquids and accordingly it is expected to apply to only specific types of systems. The relation between $m$ and $K / G$ stated by Sokolov and Novikov is based on the data analysis of non metallic and non-polymeric systems [24]. Efforts to apply this scaling to a wider group including metallic glasses or multi component systems fail [25] for obvious reasons. Polymeric materials also clearly deviate from this linear relation. Monomers follow similar correlations, but increase in the chain length in some polymers leads to the deviations [26].

The model similar to the one used here having the memory kernel with both linear and quadratic terms has been initially proposed in Ref. [20]. However the present form of the $\Gamma(t)$ ( having direct relation to the elastic constants) is only justified by including the displacement variable for the amorphous solid [11] in the theoretical description. In this regard it is useful to note that the past literature on MCT can be broadly divided into two different groups. In one approach the dynamics of a supercooled liquid is studied starting from the basic equations which apply to the liquid state or crystalline state of matter. This is the scheme we have followed in our analysis here. The set of nonlinear equations we have considered for constructing the mode coupling equations in the present context are a) the conservation laws for the mass and momentum and b) the dynamical equation for the extra slow modes for the solid like state. The resulting model linking the non ergodic behavior to the elastic properties then follows through a careful consideration of the nonlinearities in the dynamics. The present model thus extends the standard MCT in a physically relevant manner to include the solid like nature of the supercooled state. On the other hand the MCT literature also have schematic models in which ad-hoc forms of memory functions are simply assumed in order to consider different types of possible relaxation scenarios which follow from the nonlinear feed back mechanism. It is only in this respect the present model is technically similar to the schematic $\phi_{12}$ MCT studied by Götze. Finally, we have ignored here the wave 
vector dependence of the model to focus on the basic feed back mechanism. The parameter $\Delta_{o}$ has been treated as a temperature dependent parameter $\left(\sim T_{g} / T\right)$ in the model. The temperature and density dependence of the results should follow in a natural way when

such extensions of the model are considered. This calculation will require implementing the proper structure factor of the liquid in the formulation and will be considered elsewhere.

\section{ACKNOWLEDGEMENT}

MP and SPD acknowledges CSIR, India for financial support.

[1] R. Böhmer, K. L. Ngai, C. A. Angell, and D. J. Plazek, J. Chem. Phys. 99, 4201 (1993).

[2] R. Casalini, and C. M. Roland, Phys. Rev. Lett. 92, 245702 (2004).

[3] J. C. Dyre, Rev. Mod. Phys. 78, 953 (2006).

[4] V. N. Novikov, and A. P. Sokolov, Nature 431, 961 (2004).

[5] P. Bordat, F. Affouard, M. Descamps, and K. L. Ngai, Phys. Rev. Lett. 93, 105502 (2004).

[6] S. P. Das, Rev. Mod. Phys. 76, 785 (2004).

[7] D. Forster, Hydrodynamic Fluctuations, Broken Symmetry, and Correlation Functions (Benjamin, Reading, Mass., 1975).

[8] P. C. Martin, O. Parodi, and P. S. Pershan, Phys. Rev. A 6, 2401 (1972); P. D. Fleming, III, and C. Cohen, Phys. Rev. B 13, 500 (1976).

[9] S. P. Das, and R. Schilling, Phys. Rev. E 50, 1265 (1994).

[10] S.K. Ma, and G. F. Mazenko, Phys. Rev. B 11, 4077 (1975).

[11] B. Kim, Phys. Rev. A 46, 1992 (1992).

[12] J. P. Boon and S. Yip, Molecular Hydrodynamics (Dover, New York, 1991).

[13] P. C. Martin, E. D. Siggia, and H. A. Rose, Phys. Rev. A 8, 423 (1973).

[14] S. P. Das, and G. F. Mazenko, Phys. Rev. A 34, 2265 (1986)

[15] J. P. Hansen, and J. R. McDonald, Theory of Simple Liquids, 3rd ed. (Academic, London, 2005).

[16] T. R. Kirkpatrick, and J. C. Nieuwoudt, Phys. Rev. A 33, 2651 (1986); S. P. Das, Phys. Rev. A 42, 6116 (1990). 
[17] E. Leutheusser, Phys. Rev. A 29, 2765 (1984).

[18] U. Bengtzelius, W. Götze, and A. Sjölander, J. Phys. C 17, 5915 (1984).

[19] B. Kim, and G. F. Mazenko, Phys. Rev. A 45, 2393 (1992).

[20] W. Götze, and L. Sjögren, Rep. Prog. Phys. 55, 241 (1992).

[21] V. N. Novikov, Y. Ding, and A. P. Sokolov, Phys. Rev. E 71, 061501 (2005).

[22] U. Buchenau, and A. Wischnewski, Phys. Rev. B 70, 092201 (2004) and the references cited therein.

[23] L. A. Bove, C. Petrillo, A. Fontana, A. Ivanov, C. Dreyfus, and A. P. Sokolov, Physica B 385-386, 16 (2006).

[24] V. N. Novikov, and A. P. Sokolov, Phys. Rev. B 74, 064203 (2006).

[25] S. N. Yannopoulos, and G. P. Johari, Nature 442, E7-E8 (2006).

[26] A. P. Sokolov, V. N. Novikov, and Y. Ding, J. Phys.: Condens. Matter 19, 205116 (2007). 


$$
\frac{1}{2} \Sigma_{\hat{g}_{i} \hat{g}_{j}}=2 \frac{\rho}{\hat{g}_{i} u_{i}} \frac{\rho}{u_{m} \hat{g}_{j}}+4 \frac{\rho}{\hat{g}_{i} \rho} \frac{\rho}{\rho \hat{g}_{j}}
$$

FIG. 1: Relevent one loop diagrams which contribute to the effective viscosity as non-linear corrections.

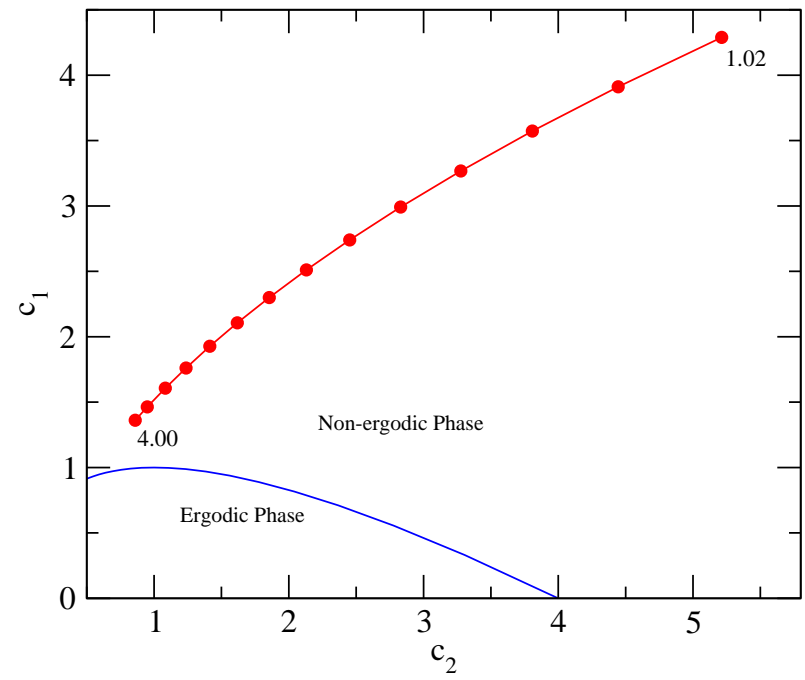

FIG. 2: $c_{1}$ vs. $c_{2}$ and the solid line displays the dynamic transition line. The circles (joined with solid line to show continuity) shown in the nonergodic phase correspond to the states for which the results of fig. 5 and 7 are obtained $\left(\Delta_{0}=1.7\right)$. These circles correspond to different values of the ratio $K / G$ of bulk and shear moduli with the adjacent number to a circle indicating the corresponding value of $\mathrm{K} / \mathrm{G}$. 


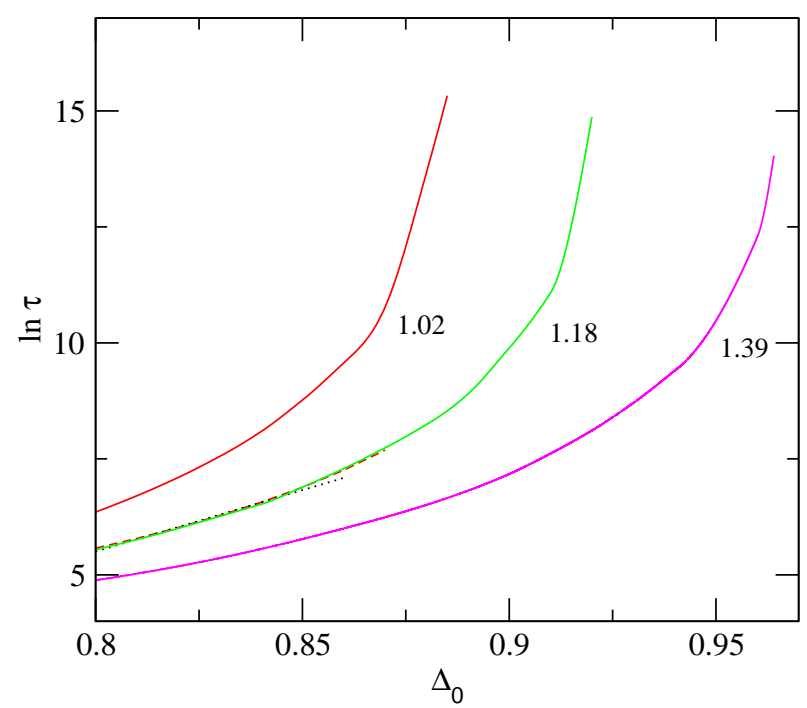

FIG. 3: Log of relaxation time $\tau$ in units of $\Gamma_{0} /\left(\rho_{o} c_{L}^{2}\right)$, vs $\Delta_{0}$ (see text). The constant $K / G$ value corresponding to each curve is also displayed adjacent to it in the figure. The dashed and dotted lines are, respectively, the power law and exponential fits to the corresponding curve.

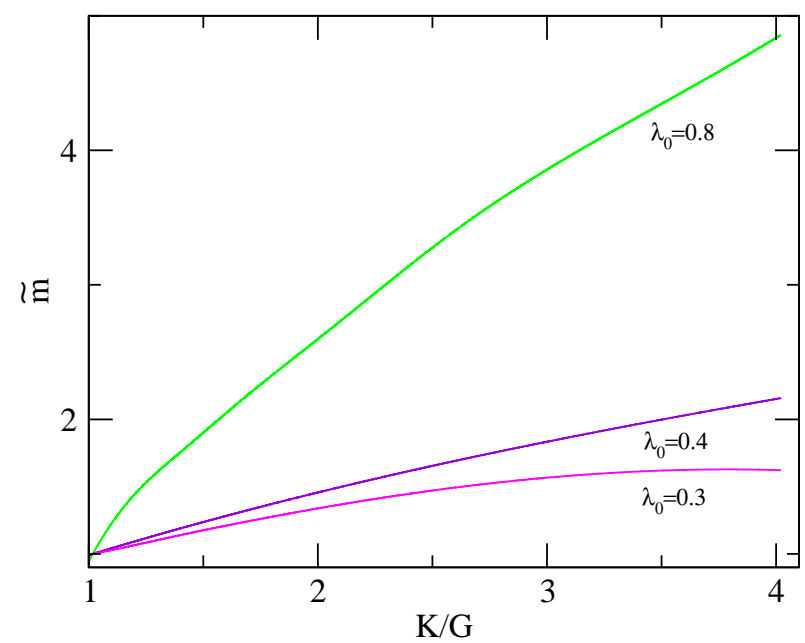

FIG. 4: The inverse slope $\tilde{m}$ (see text) vs ratio $K / G$ of bulk and shear moduli at different $\lambda_{0}$ values, displayed in the figure. 


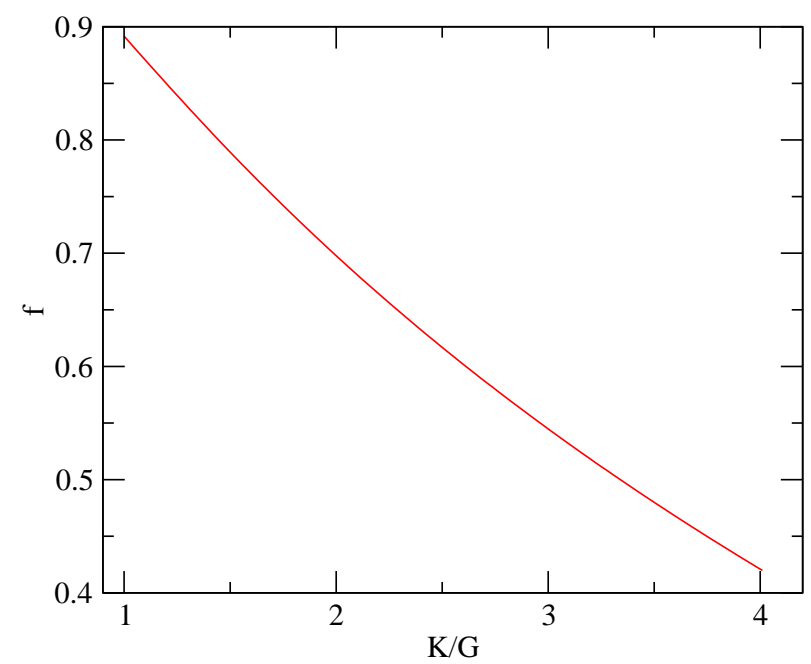

FIG. 5: Non ergodicity parameter $f$ vs the ratio $K / G$ of bulk and shear moduli from the present model $\left(\Delta_{0}=1.7\right)$.

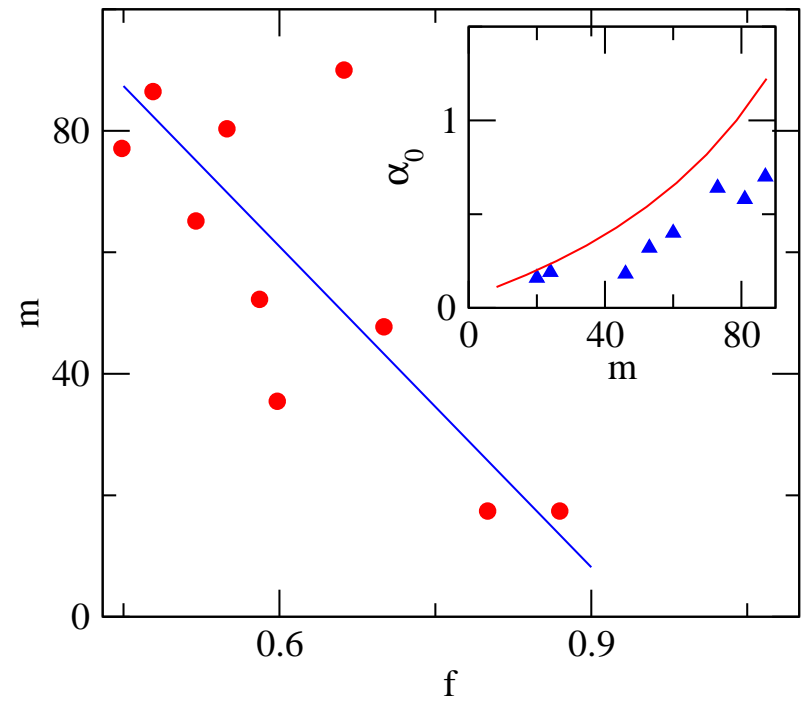

FIG. 6: Experimental data (points) and best fit (line) to the experimental data Ref. [21] linking fragility index $m$ with $f$. The inset shows $\alpha_{o}=(1-f) / f$ vs fragility index $m$ ( solid line) (using the experimental best fit), X-ray Brillouin scattering data ( solid triangles) 22]. 


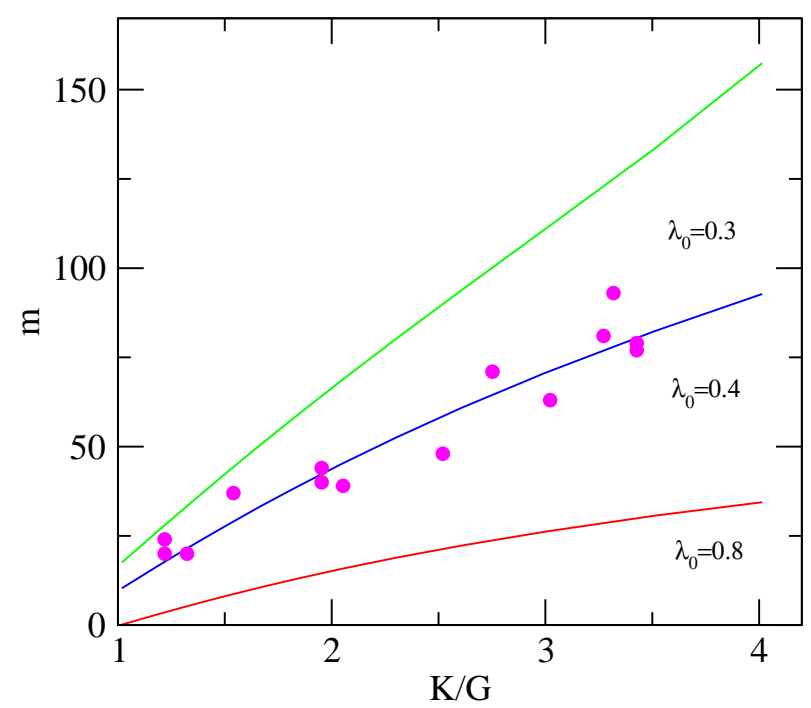

FIG. 7: Fragility index $m$ vs ratio $K / G$ of bulk and shear moduli from the present model at different $\lambda_{0}$ values (solid lines) $\left(\Delta_{0}=1.7\right)$, experimental data of Ref. [4] (points).

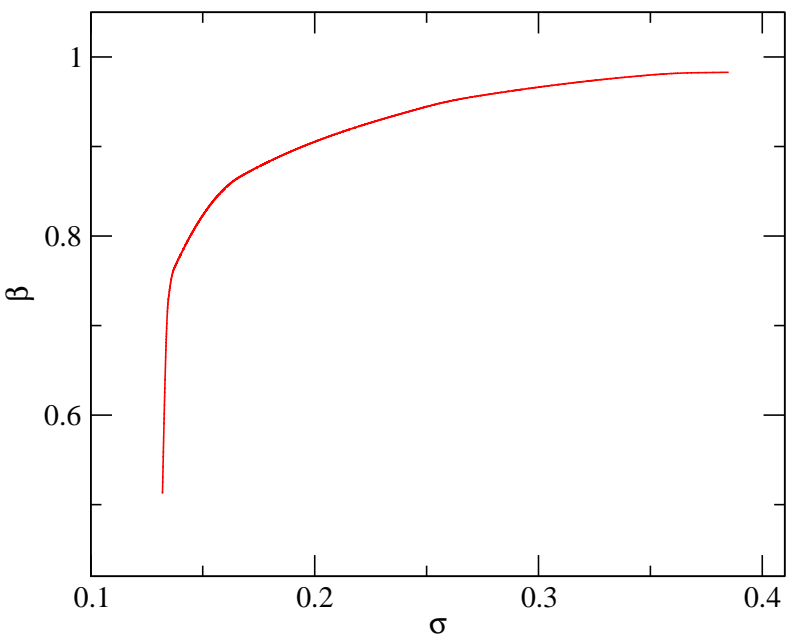

FIG. 8: Stretched exponential exponent $\beta$ vs Poisson's ratio $\sigma\left(\Delta_{0}=0.885\right)$. 


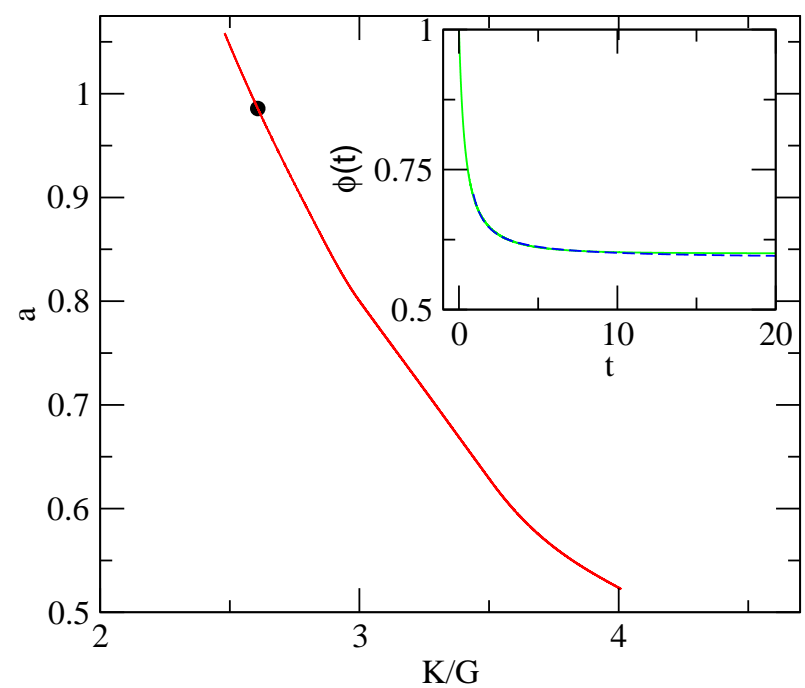

FIG. 9: Power law exponent $a$ vs the ratio $K / G$ of bulk and shear moduli $\left(\Delta_{0}=1.7\right)$. Inset shows normalized density correlation function $\phi(t)$ vs $t$ in units of $\Gamma_{0} /\left(\rho_{o} c_{L}^{2}\right)$ (see text). The dashed curve is the power law fit with the exponent value shown as a dot on the main figure. 